\title{
EED-associated overgrowth in a second male patient
}

\author{
Ana SA Cohen ${ }^{1,2}$ and William T Gibson ${ }^{1,2}$ \\ Following our discovery that constitutional mutations in EED can cause overgrowth, we screened our cohort of patients with \\ Weaver-like features for mutations in this gene. Here we describe a second patient with a different, rare and de novo mutation in \\ $E E D$. Phenotypic overlap with our first case of EED-associated overgrowth is significant. Now that we have found two unrelated \\ families of different ethnicities, with a similar rare phenotype, both associated with de novo mutations in this member of the \\ PRC2 complex, we are confident that EED is indeed a novel overgrowth gene.
}

Journal of Human Genetics (2016) 61, 831-834; doi:10.1038/jhg.2016.51; published online 19 May 2016

\begin{abstract}
INTRODUCTION
Weaver syndrome (MIM \#277590) is a 'classical' overgrowth syndrome that shares phenotypic overlap with Sotos syndrome (MIM \#117550). ${ }^{1}$ Constitutional mutations in NSD1 cause Sotos syndrome; $^{2}$ constitutional mutations in EZH2 cause Weaver syndrome. ${ }^{3,4}$ Recently, we described a patient suspected clinically to have Weaver syndrome but whose features were caused by a rare de novo mutation in EED. ${ }^{5}$ EED partners with EZH2 in the polycomb repressive complex 2 (PRC2) that maintains gene silencing (Figure 1a); ${ }^{6}$ binding to EED is essential for proper EZH2-mediated methyltransferase activity. ${ }^{7}$ Here we report a man with overgrowth, facial dysmorphism and intellectual disability, in whom we identified a different mutation in EED that is both rare and de novo (Figure 1b). He shows significant phenotypic overlap with our original patient. ${ }^{5}$ This is the second report of overgrowth and characteristic dysmorphism associated with a constitutional mutation in EED.
\end{abstract}

\section{CLINICAL REPORT}

Birth and early years

Our proband required forceps-assisted delivery after an uncomplicated term pregnancy (42 weeks by dates). His parents are non-consanguineous Caucasians and have younger healthy twin sons; there is no family history of overgrowth. The father was 36 and the mother 32 years at conception. Ultrasound at the beginning of the third trimester identified macrosomia. Birth weight was $4366 \mathrm{~g}$, length $54.6 \mathrm{~cm}$ and head circumference $37.2 \mathrm{~cm}$ (Supplementary Figures 1 and 2 and Supplementary Table 1$)$. Apgar scores were $3^{(1 \mathrm{~min})}$ and $4^{(5 \mathrm{~min})}$. He had respiratory distress and mild jaundice. An umbilical hernia developed 1 week after birth. Developmental delay was apparent early: he could only say one word by 14 months and 2 words by 19 months. At 17 months, he could feed himself and hold himself up; he crawled a few weeks later. When standing, his legs and Achilles tendons were stiff and he stood primarily on his toes; physiotherapy improved his range of movement. Karyotype was normal (46, XY), and he was referred to Medical Genetics where his delayed motor skills, cognitive difficulties, large size and dysmorphic facies (Figures $2 \mathrm{a}-\mathrm{c}$ and $\mathrm{f}$ ) suggested Weaver syndrome. At 20 months, he took four steps alone. At 22 months, he got casts for heel-cord lengthening and said his third word. At 24 months, he could walk unassisted. At 26 months, he started learning sign language and at 30 months he could say 3 more words. He could also go up and down stairs unassisted.

\section{Childhood}

At 5 years, some asymmetry of the skull was noted and X-rays revealed a bone age of 8 years. At 6 years 1 month, his verbal scores remained equivalent to age 2.5 years. Insulin-like growth factor 1 levels were normal at age 6 years 5 months. He could ride a bike at age 7 years. By the time he reached third grade (around age 8 years), his speech was much improved and he interacted socially with his peers. At 8 years 8 months (Figure 2g), his overall IQ was 52 (verbal IQ 64, performance IQ 47; WISC-III): he could not read or recognize numbers but could count objects, and specific weaknesses were noted in problem solving and memory (except for visual memory). Caregivers found him to be socially interactive and very personable. Slowness with upper extremity motor skills (attributed to dyspraxia) made it hard for him to write, though he could copy drawings and write his own name. Hypotonia also contributed to coordination and balance difficulties, with marked pronation of the feet and bent knees, in turn affecting the posture of his hips and back. Other biomechanical variations (rigidity of the first ray bilaterally and hypermobility of the fourth and fifth rays, and limited midtarsal locking bilaterally) also affected his joint stability. At 8 years 10 months, reduced range of motion in large joints was observed. Physical therapy (movement and gait training) continued. At 9 years 4 months, dental examination revealed excess overbite and overjet, deep anterior bite with

\footnotetext{
${ }^{1}$ Department of Medical Genetics, University of British Columbia, Vancouver, British Columbia, Canada and ${ }^{2}$ Child and Family Research Institute, British Columbia Children's Hospital, Vancouver, British Columbia, Canada

Correspondence: ASA Cohen, Department of Medical Genetics, University of British Columbia, 950 West 28th Avenue, A4-151, bay 17, Vancouver, British Columbia, Canada V5Z 4H4.

E-mail: acohen@cfri.ca

Received 24 December 2015; revised 30 March 2016; accepted 13 April 2016; published online 19 May 2016
} 


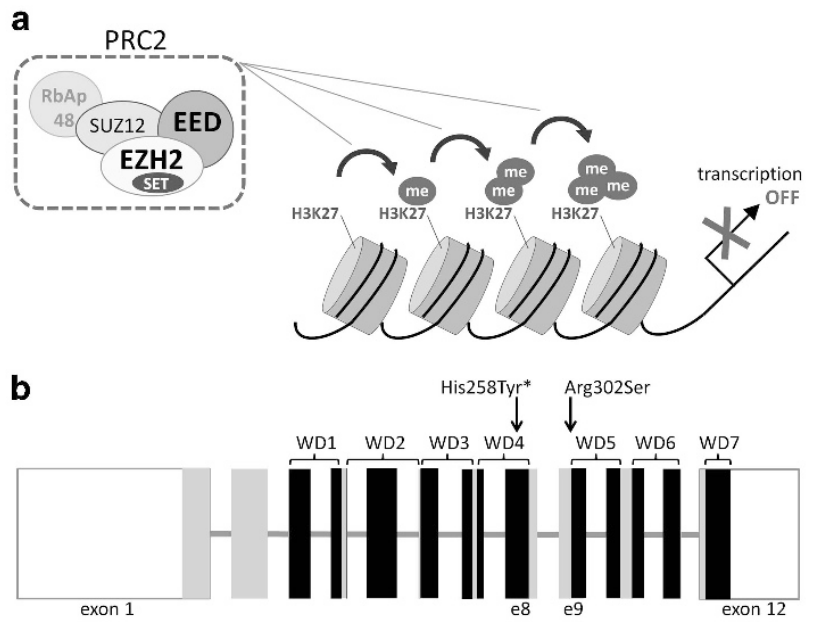

Figure 1 Schematic of human EED and its role within the polycomb repressive complex 2 (PRC2). (a) Schematic representation of PRC2's role. EED is required (along with EZH2 and SUZ12) for proper histone methyltransferase activity mediated by the SET domain of EZH2. Within PRC2, EZH2 can add up to 3 methyl groups (me) to lysine 27 on the histone 3 tail (H3K27). This is done in a sequential manner and shuts off transcription, leading to repression of gene expression. Disruption of EED is thought to disturb this PRC2-mediated histone methyltransferase activity. (b) Human EED is represented. Each rectangle represents one exon. Exon size is represented to scale, while intronic distances are not to scale. White (open) rectangles represent non-coding UTRs and gray rectangles represent coding exons (NM_003797.4). EED protein contains 441 amino acids (NP_003788.2) and seven WD repeats, represented here in black according to UniProt (075530) coordinates. The two constitutional mutations in EED associated with overgrowth are shown (current case indicated by asterisk).

impingement, and a vertical facial growth pattern with moderate mandibular retrognathia. Treatment included headgear and retainers for several years. By the fifth grade (around 10 years of age), his speech had improved and was rated as clear to unfamiliar individuals approximately $80 \%$ of the time. At 10 years 4 months, X-rays of the spine showed thoracolumbar scoliosis $\left(18^{\circ}\right)$, and X-rays of the hand and wrist again showed advanced bone age (consistent with 12 years 6 months) and moderate osteopenia. Abdominal ultrasound was normal. His scores on the Vineland (measured at 12 years 3 months) assessed his communication and daily living skills to be at a $6+$ year level, gross motor and fine motor skills at a 4-5 year level, adaptive skills at a $6+$ year level, and social skills at $7+$ year level. He had chronic constipation requiring Citrucel and high fiber cereals. Notable dysmorphisms included large hands with long slender fingers (middle finger length $9.5 \mathrm{~cm}$, total hand length $20 \mathrm{~cm}$ ) and large slender feet (total length $28 \mathrm{~cm}$ ), macrocephaly, almond-shaped palpebral fissures and bifid uvula. His rate of growth had slowed, with measurements on the 75th percentile (height $159.7 \mathrm{~cm}$, weight $46 \mathrm{~kg}$ and head circumference $57.2 \mathrm{~cm}$; Supplementary Figure 3). Weaver syndrome remained the most plausible recognized diagnosis for his phenotype (Figure 2h). At 14 years 2 months, reading was equivalent to a first grade level.

\section{Trauma, surgery and recovery}

At age 15 years 6 months, the proband suffered neck trauma: he did a forward roll in gymnastics class and had immediate onset of gait ataxia, with numbness and weakness of his extremities. MRI revealed spinal cord compression at the occipitocervical junction. Flexion extension radiographs revealed significant $\mathrm{C} 1-\mathrm{C} 2$ instability, a substantially increased atlanto-dens interval and assimilation of the atlas. Surgical treatment included suboccipital craniectomy, C1 and C2 laminectomies, and lysis of dural band adhesions. Follow-up examination at two and half months after surgery showed functional recovery; physical therapy eventually corrected his residual cervical misalignment.

\section{Adult years}

Brain MRI at 22 years of age showed no evidence of impingement on the medulla or proximal cervical cord following surgical fusion and posterior decompression. At the latest examination (age 30 years 4 months), he was doing well and communicated verbally equivalent to a first grade level. He enjoyed meeting new people and had a good sense of humor. His adult height was $191 \mathrm{~cm}$ (>97th percentile), weight $93.4 \mathrm{~kg}$ (90-95th percentile) and head circumference $61 \mathrm{~cm}$ ( $>90$ th percentile). His stance remained slightly forward, with hips and knees flexed. Range of movement of certain joints was restricted: he was unable to reach his arms up over his head or to bend down to tie his shoes, with his heel cords remaining very tight. His fingernails and toenails were very fragile. Further features are shown in Figures 2i-l.

\section{SANGER SEQUENCING AND RESULTS}

After informed consent, Sanger screening of EZH2 and NSD1 in the proband was negative for rare variants. Following our discovery that a constitutional mutation in EED caused Weaver-like features in another proband, ${ }^{5}$ we screened all coding exons of EED (Figure 1b) by Sanger Sequencing in this proband. We identified a c.772 C $>\mathrm{T}$ missense variant (c.1238C $>\mathrm{T}$ in the full-length messenger RNA sequence NM_003797.3) that was absent from his parents and siblings (Figures 2d,e). This variant, predicted to convert histidine residue 258 to tyrosine (p.His258Tyr), is not reported in the single nucleotide polymorphism database or COSMIC databases nor in the exome variant server, and was predicted damaging by both PROVEAN and SIFT (http://provean.jcvi.org/genome_submit_2.php?species=human). It is thus both a novel variant and a de novo mutation.

\section{DISCUSSION}

This is the second report of a rare de novo constitutional mutation in $E E D$ associated with overgrowth, intellectual disability and dysmorphic features. EED contains seven WD domains that interact physically with other proteins, particularly EZH2. ${ }^{6-9}$ Our patient's mutation (encoding p.His258Tyr) is located in a highly conserved region ${ }^{8,10}$ within the fourth WD domain ${ }^{11}$ (Figure 1b). Of interest, a somatic mutation located at the following amino acid (Ser259Phe) has been described in acute lymphoblastic leukemia, ${ }^{12}$ and two loss-of-function mutations $\left(e s c^{9}\right.$ and $\left.e s c^{1}\right)$ have been identified at nearby residues in the Drosophila orthologue (corresponding to Met256Lys and Leu260Arg in human EED). ${ }^{13,14}$ These independent lines of evidence support functional importance of this protein domain.

Furthermore, in vitro assays of the Drosophila esc ${ }^{9}$ variant showed that the mutated protein did not bind $\mathrm{E}(\mathrm{Z})$, the orthologue of $\mathrm{EZH} 2$, as efficiently as wild type, ${ }^{8}$ and Ketel et al. ${ }^{15}$ showed that complexes harboring the $\operatorname{esc}^{9}$ mutation have reduced histone methyltransferase activity. In addition, Sewalt et al. ${ }^{6}$ showed that all WD domains in human EED must remain intact for proper binding and interaction with EZH2, and Montgomery et al. ${ }^{9}$ showed that deletion of individual WD domains disrupts PRC2-mediated H3K27 methylation in mouse embryonic stem cells. Together, these data suggest that disruption of EED's WD domains reduces PRC2-mediated H3K27 methylation by separating EED from EZH2. Consistent with this hypothesis, the nearby residue Met256 is located on the outer surface of EED, at a 

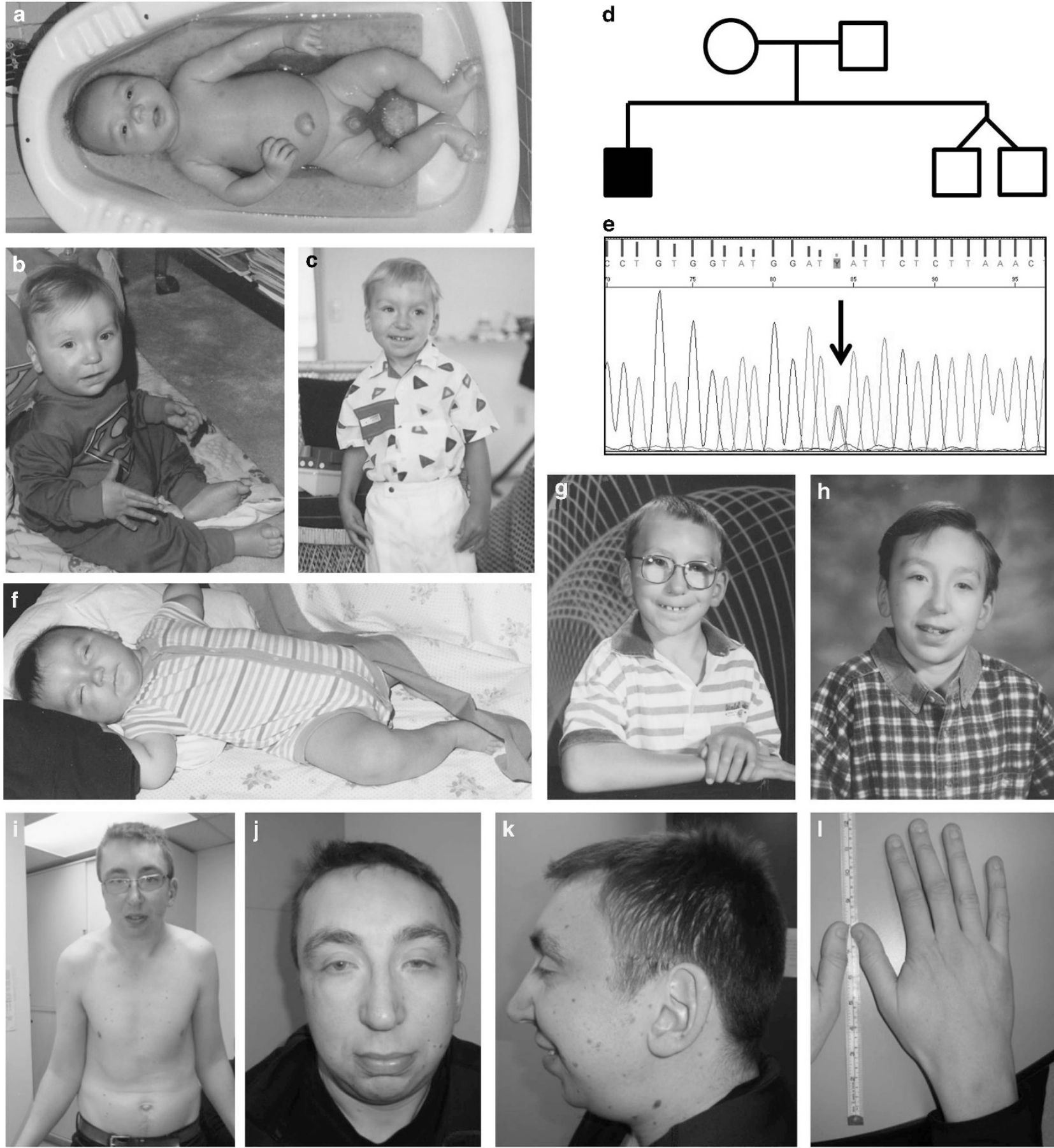

e
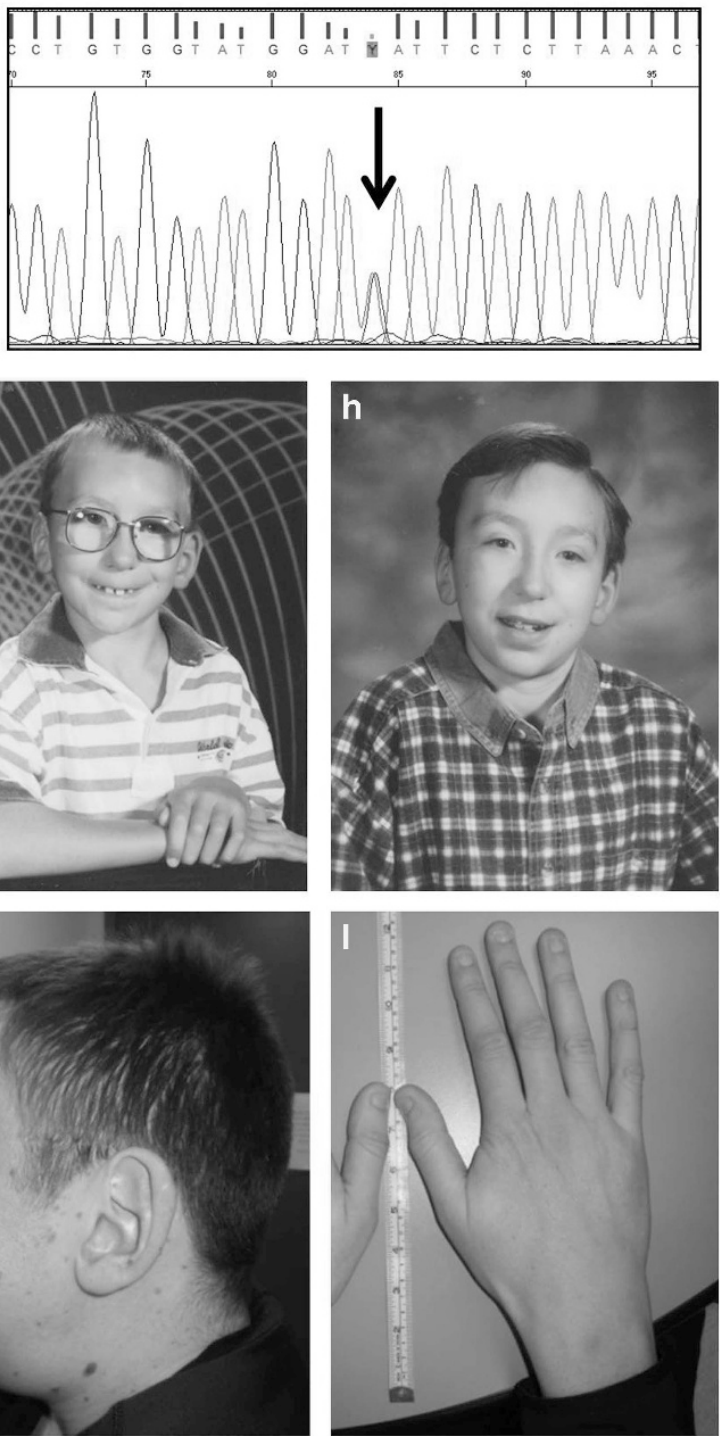

Figure 2 Second proband with a constitutional mutation in EED. (a-c) Photographs of the proband at age 6 weeks (a), 6 months (b) and 2 and a half years (c) show that early features were consistent with Weaver syndrome. Note the rounded face, macrocephaly, retrognathia with 'stuck-on' chin, long and slender nose, and large low-set ears. (d) Pedigree of the family showing that the proband is the only affected individual. (e) Sanger sequencing identified a de novo c.1238C $>\mathrm{T}$ (p. His258Tyr) mutation in EED, exclusive to the affected proband. ( $\mathbf{f}-\mathbf{I})$ Photographs of the proband at various ages illustrate an evolving phenotype. Typical features of Weaver syndrome observed in early life (f: 7 months of age) remained apparent through childhood (g: 8 years and $\mathbf{h}: 12$ years). Recent photographs at age 30 years and 4 months (i-I) show dysmorphic features in adulthood. The main features include deep-set eyes, large low-set ears, prominent nasal root and nasal bridge with bulbous nasal tip, and retrognathia with a prominent crease between the lower lip and the chin (j, k). His thorax is narrow and pivoted forward on his hips slightly, and slight kyphosis is apparent (i). He also has numerous pigmented nevi across his chest (i) and face (k). The proband's right hand shown at 30 years and 4 months (I) is unusually large, measuring $23.5 \mathrm{~cm}$. The wrist is broad, fingers are long and slender with long phalanges and very thick skin over the knuckles, and fingernails are fragile and paper-thin. A full color version of this figure is available at the Journal of Human Genetics journal online. 
prime position to interact with partner proteins. ${ }^{8,11}$ Thus, mutations in $E E D$ could lead to overgrowth via similar molecular mechanisms to EZH2 mutations, which appear to reduce H3K27 methylation, ${ }^{16}$ potentially causing derepression of Hox genes during embryonic development. ${ }^{7,13,14,17}$ The pathophysiological mechanism is not yet understood in detail and will require further investigation.

Based on the evidence discussed here and in Cohen et al. ${ }^{5}$ and the fact that we have identified two different rare and de novo mutations in EED that are associated with overgrowth, intellectual disability and characteristic dysmorphism, we conclude that these are truly pathogenic variants and that we have successfully identified a new overgrowth gene. Both of our patients presented with Weaver-like features at an early age, including a rounded face with a 'stuck-on' chin, overgrowth and intellectual disability (Supplementary Table 1). It has been well established that many features attenuate with age in Weaver syndrome patients, ${ }^{4}$ whereas these two individuals with EED mutations remain very dysmorphic in adulthood and have more severe skeletal perturbations, as well as restricted joint movement and unusually large hands. $E E D$-associated overgrowth does not consistently remain above the 95th percentile for stature and weight throughout childhood and adolescence (Supplementary Figure 3), though adult height of both patients was above the 95th percentile (Supplementary Table 1). Predisposition to hematological and other malignancies is known to occur in Weaver syndrome; ${ }^{1,4,16}$ to date, neither patient with a de novo EED mutation has developed neoplasia, but the large number of cutaneous nevi in this patient suggests the possibility of precancerous lesions. We will need to identify additional patients (particularly older adults) and follow them longitudinally to get better data on constitutional cancer predisposition, if any, conferred by mutations in EED. Additional cases will also allow us to characterize the full phenotypic spectrum of EED-associated overgrowth.

\section{CONFLICT OF INTEREST}

The authors declare no conflict of interest.

\section{ACKNOWLEDGEMENTS}

We thank the family for their enthusiastic participation in our study, and we gratefully acknowledge the staff of the sequencing core at the Centre for Molecular Medicine and Therapeutics (UBC) for carrying out Sanger sequencing post-PCR. We also thank the Centre for Applied Neurogenetics (UBC) for confirming family relationships on our samples. This work was supported by the CIHR (Operating Grants PCN102990, PCN110794 and
MOP119595), and by Clinician Scientist salary awards to WTG (CIHR and Child and Family Research Institute). ASAC holds a Doctoral Grant from the Fundação para a Ciência e a Tecnologia (Portugal/EU).

1 Tatton-Brown, K. \& Rahman, N. The NSD1 and EZH2 overgrowth genes, similarities and differences. Am. J. Med. Genet. C 163C, 86-91 (2013).

2 Kurotaki, N., Imaizumi, K., Harada, N., Masuno, M., Kondoh, T., Nagai, T. et al. Haploinsufficiency of NSD1 causes Sotos syndrome. Nat. Genet. 30, 365-366 (2002).

3 Gibson, W. T., Hood, R. L., Zhan, S. H., Bulman, D. E., Fejes, A. P., Moore, R. et al. Mutations in EZH2 cause Weaver syndrome. Am. J. Hum. Genet. 90, 110-118 (2012).

4 Tatton-Brown, K., Murray, A., Hanks, S., Douglas, J., Armstrong, R., Banka, S. et al. Weaver syndrome and EZH2 mutations: Clarifying the clinical phenotype. Am. J. Med. Genet. A 161A, 2972-2980 (2013).

5 Cohen, A. S. A., Tuysuz, B., Shen, Y., Bhalla, S. K., Jones, S. J. M. \& Gibson, W. T. A novel mutation in EED associated with overgrowth. J. Hum. Genet. 60, 339-342 (2015).

6 Sewalt, R. G., van der Vlag, J., Gunster, M. J., Hamer, K. M., den Blaauwen, J. L., Satijn, D. P. et al. Characterization of interactions between the mammalian polycombgroup proteins Enx1/EZH2 and EED suggests the existence of different mammalian polycomb-group protein complexes. Mol. Cell. Biol. 18, 3586-3595 (1998).

7 Cao, R. \& Zhang, Y. SUZ12 is required for both the histone methyltransferase activity and the silencing function of the EED-EZH2 complex. Mol. Cell 15, 57-67 (2004).

8 Tie, F., Furuyama, T. \& Harte, P. J. The Drosophila Polycomb Group proteins ESC and E (Z) bind directly to each other and co-localize at multiple chromosomal sites. Development 125, 3483-3496 (1998).

9 Montgomery, N. D., Yee, D., Montgomery, S. A. \& Magnuson, T. Molecular and functional mapping of EED motifs required for PRC2-dependent histone methylation. J. Mol. Biol. 374, 1145-1157 (2007).

10 Schumacher, A., Lichtarge, O., Schwartz, S. \& Magnuson, T. The murine Polycombgroup gene eed and its human orthologue: functional implications of evolutionary conservation. Genomics 54, 79-88 (1998).

11 Han, Z., Xing, X., Hu, M., Zhang, Y., Liu, P. \& Chai, J. Structural basis of EZH2 recognition by EED. Structure 15, 1306-1315 (2007).

12 Zhang, J., Ding, L., Holmfeldt, L., Wu, G., Heatley, S. L., Payne-Turner, D. et al. The genetic basis of early T-cell precursor acute lymphoblastic leukaemia. Nature 481, 157-163 (2012).

13 Sathe, S. S. \& Harte, P. J. The Drosophila extra sex combs protein contains WD motifs essential for its function as a repressor of homeotic genes. Mech. Dev. 52, 77-87 (1995).

14 Ng, J., Li, R., Morgan, K. \& Simon, J. Evolutionary conservation and predicted structure of the Drosophila extra sex combs repressor protein. Mol. Cell. Biol. 17, 6663-6672 (1997).

15 Ketel, C. S., Andersen, E. F., Vargas, M. L., Suh, J., Strome, S. \& Simon, J. A. Subunit contributions to histone methyltransferase activities of fly and worm polycomb group complexes. Mol. Cell. Biol. 25, 6857-6868 (2005).

16 Cohen, A. S. A., Yap, D. B., Lewis, M. E. S., Chijiwa, C., Ramos-Arroyo, M. A., Tkachenko, N. et al. Weaver Syndrome-Associated EZH2 Protein Variants Show Impaired Histone Methyltransferase Function In Vitro. Hum. Mutat. 37, 301-307 (2016).

17 Khan, A. A., Lee, A. J. \& Roh, T. Y. Polycomb group protein-mediated histone modifications during cell differentiation. Epigenomics 7, 75-84 (2015).

Supplementary Information accompanies the paper on Journal of Human Genetics website (http://www.nature.com/jhg) 